\title{
Cell based therapy in Parkinsonism
}

\author{
Johannes PJM de Munter ${ }^{1,2^{*}}$, Chongsik Lee ${ }^{3}$ and Erik Ch Wolters ${ }^{1,4}$
}

\begin{abstract}
Parkinson's disease (PD) is a synucleinopathy-induced chronic progressive neurodegenerative disorder, worldwide affecting about 5 million humans. As of yet, actual therapies are symptomatic, and neuroprotective strategies are an unmet need. Due to their capability to transdifferentiate, to immune modulate and to increase neuroplasticity by producing neurotrophic factors, adult stem cells (ASC) might fill this gap. Preclinical research in 6-hydroxydopamine (6-OHDA) and/or 1-methyl-4-phenyl-1,2,3,6-tetrahydropyridine (MPTP) lesioned animals established persistent improvements of motor behavior after ASC-treatment. Histological/histochemical measurements in these animals evidenced an intracerebral applied ASC-induced increase of Tyrosine Hydroxylase-positive $\left(\mathrm{TH}^{+}\right)$cells with increased striatal dopamine levels, suggesting cell rescue. Likewise, clinical experience with subventricular applied ASCs in PD patients, although limited, is encouraging, evidencing neurorescue especially during the early phase of the disease. In multiple system atrophy (MSA) or progressive supranuclear palsy (PSP) patients, though, only marginal reduced progression of natural progression could be established after subventricular or intravasal ASC implantations.
\end{abstract}

Keywords: Adult stem cells, Parkinson's disease, Multiple system atrophy, BDNF, GDNF, Expanded MSC, Preclinical

\section{Introduction}

Parkinson's disease (PD) is the most common chronic progressive neurodegenerative disorder after Alzheimer's disease [1], world-wide affecting nearly 5 million people aged 50 years or more, and expected to double over the next 20 years [2]. It comes with a twofold higher mortality rate, mainly due to pneumonia, shortening life expectancy with nearly 10 years $[3,4]$. The result of the $\alpha$-synucleinopathic degeneration of the nervous system, starting in the peripheral nervous system and lower brainstem and progressively extending over the upper brainstem and neocortex, symptomatology in PD comprises dysfunctions of the whole nervous system. It may start with a range of non-motor symptoms such as disorders of the autonomic nervous system, olfaction, sleep, mood and subtle cognitive deterioration, before a degeneration of the dopamine producing cells in the upper brainstem (nigral substance) may manifest with motor parkinsonism, the clinical hallmark of this disease, and way before involvement of the neocortex induces dementia [5]. PD is mainly recognized when first symptoms of motor parkinsonism (hypokinesia, bradykinesia, rigidity, tremor and the loss of postural

\footnotetext{
* Correspondence: h.demunter@hetnet.nl

'Department of Neurosciences University Maastricht, Maastricht, The Netherlands

${ }^{2}$ Amarna Stem Cells Group, Maastricht, The Netherlands

Full list of author information is available at the end of the article
}

reflexes) develop as the result of the loss of the majority of the dopaminergic neurons of the pars compacta of the substantia nigra with a striatal dopaminergic depletion of over $80 \%$ [6]. As of yet, treatment in PD is based on the pulsatile (oral) or continuous (subcutaneous, intrajejunal) suppletion of the striatal dopamine deficiency with dopamine agonists and/or the dopamine precursor levodopa, mostly in combination with a peripheral dopa decarboxylase inhibitor and/or in combination with inhibitors of mono-amine oxidase $\mathrm{B}$ (MAO-B) and/ or catechol-O-methyl transferase (COMT), in order to restore striatal dopaminergic denervation [7].

Actual therapy only symptomatically affects motor parkinsonism, though. Therapies affecting non-motor symptomatology, and above all protective or restorative treatments are unmet needs in PD. In order to reach these needs, recently, experiments with cell based therapies to rescue or replace dopamine-secreting cells, or with cells able to secrete paracrine factors modulating brain tissue repair were initiated [8-12].

In this review, these experimental stem cell based therapeutic strategies will be discussed. As the application of embryonic stem cells and induced pluripotent stem cells comes with an unacceptable risk of tumor induction [13-16], this review will only cover experiments dealing with expanded, whether or not genetically 
modified, autologous or allogenic bone marrow-derived and/or neural progenitor stem cells.

\section{Adult stem cells (ASC)}

Adult stem cells comprise mesenchymal stem cells (MSCs), hematopoietic stem cells (HSCs) and ectodermal stem cells (ESCs). The majority of the cited preclinical and clinical studies use expanded and/or induced mesenchymal stem cells.

Re-implanted adult autologous stem cells, easily harvested out of the iliac crest and whether or not expanded, as a rule, will migrate towards diseased tissue, a phenomenon called homing $[17,18]$. Those stem cells have the potency to modulate immune responses $[19,20]$ and to both transdifferentiate into target cells in order to replace damaged cells [21-24], and secrete paracrine (trophic) factors relevant for cell protection and cell repair by the inhibition of apoptotic pathways [25-27]. So, even before differentiation [28,29], mesenchymal stem cells, might express brain-derived neurotrophic factor (BNDF), glial cell-derived neurotrophic factor (GDNF) and stromal-derived factor (SDF-1 $\alpha$ ). BDNF is shown to have a neuroprotective effect on cultured rodent neurons via the $\mathrm{Pl}_{3}$ kinase/Akt pathway by inhibiting neural death initiated by trophic factor withdrawal or by the exposure to nitric oxide [30]. GDNF provides neural protection against proteasome inhibitor-induced dopamine neuron degeneration [31], although its biological effect on the clearance of mature formed $\alpha$-synuclein aggregation could not be observed, probably due to its short duration of administration [31]. SDF- $1 \alpha$, in low doses, promotes dopamine release from 6-OHDA-exposed PC12 cells (cell line derived from a pheochromocytoma), presumably by preservation and enhanced survival of these cells, as these phenomena are blocked by administration of anti-SDF-1 $\alpha$ antibodies [32]. A high concentration of SDF-1 $\alpha$, however, rather enhances apoptosis [33]. SDF- $1 \alpha$ acts through CXCR4 (chemokine receptor type 4) resulting in a down regulation of caspase-3 and an activation of the PI3/Akt pathway [34]. SDF-1 $\alpha$ also enhances the survival of neural progenitor cells through the receptors CXCR7 and CXCR4 by up regulation of the ERK1/2 (Mitogen-Activated Protein kinase 3) endocytotic signaling pathway [35].

The route of administration (intravasal, intraparenchymal) during the re-implantation of the stem cells seems to have a major impact on the specific transdifferentiation and/or secretion patterns of them, as the actual environment influences the further developments of these cells. However, by inducing stem cells before re-implantation it is also possible to induce these developments already in vitro. Indeed, by exposing these cells to trophic factors, including epidermic growth factor (EGF) and basic fibroblast growth factor (bFGF) [36], by transducing them with a viral vector, and/or by binding them to pharmacologically active microcarriers, containing trophic factors such as NT-3 (Neurotrophin-3) [37], it is possible to further differentiate the stem cells, prior to the re-administration to the target organs $[38,39]$. So, in vitro, adult stem cells may be predisposed to differentiate into dopamine producing cells $[21,40]$, thus offering a potential alternative for dopamine substitution therapies, or into cells secreting neuroprotective and/or neurorestorative trophic factors, thus protecting for dopaminergic cell death respectively stimulating neurorestoration [41].

Due to the lung trap [42] and the blood-brain-barrier $[43,44]$, intravasal application of stem cells for disorders of the central nervous system is suggested less effective as compared to intracerebral administration $[23,45]$.

\section{Preclinical experience with ASC in motor parkinsonism}

In experimental animals, motor parkinsonism (but not PD) might be induced by intra-nigral 6-hydroxydopamine (6-OHDA) as well as by subcutaneous carbobenzoxy-leuleu-leucinal (MG-132) and/or subcutaneous or intravasal 1methyl-4-phenyl-1,2,3,6-tetrahydropyridine (MPTP) [46,47]. MPTP models have the advantage that the lesions are induced through a less invasive (subcutaneous or intravasal) route of administration as compared with the stereotactic surgical approach when applying 6-OHDA. To optimally investigate neural protection, the non-human primate MPTP model seems to be preferred [46]. Tables 1 and 2 present the results of preclinical interventions using cell-based strategies in the 6-OHDA, MPTP and the proteasome inhibitor MG-132 animal models. In all the presented animal studies immune suppression was used to prevent rejection of the cells. However, the use of immune suppression may influence not only the behavior of the Stem Cells [48] but also the severity of the lesions in animal models $[49,50]$.

As might be appreciated, in the studies reviewed in Tables 1 and 2, the following stem cells were used:

- Expanded bone marrow-derived mesenchymal cells (of the mesodermal lineage) of rat [32,37,51-53], mouse [54] or human origin [55-58];

- Expanded and enriched, or epigenetically induced bone marrow-derived mesenchymal stem cells $[37,51,55,56]$, enabling functional development before actual transplantation;

- Expanded neural stem cells of the ectodermal lineage, derived from the subventricular zone of the rat [59] or from a cultivated fetal human cell line $[60,61]$.

But for three studies with intravenous [32,54] and/or intranasal [53] application, all stem cells are applied intranigral and/or intrastriatal.

Outcome measures comprised motor behavior (rotational behavior) as well as histological/histochemical measures. The main findings on the rotational motor 
Table 1 Placebo-controlled stem cell applications in rodent animal models of parkinsonism

Striatal 6-OHDA lesioned rats

Authors animals
(Sprague-Dawley
rats)

Bouchez, Sensebe et al. [51]

Female rats

riMSCs

Wang, Yasuhara

et al. [32]

Female rats

Danielyan, Schafer et al. [53]

Female rats

Blandini, Cova

et al. [58]

hMSCs

Male rats

rMSCs
Product expanded allogenic ASCs Cyclosporin A

4/35 days

2. Intrastriatal saline $(n=7)$

3. Intrastriatal $1.8 \times 10^{5}$ $\operatorname{rMSCs}(n=7)$

4. Intrastriatal $1.8 \times 10^{5}$ riMSCs $(n=7)$

$2 \mathrm{hr} / 28$ days

1. Intravenous saline $(n=7)$

2. Intravenous $10^{7}$ fibroblasts $(n=6)$

3. Intravenous $10^{7} \mathrm{rMSCs}$ $(n=6)$

7,9/110-136 days

Intranasal saline day 7 and 9 $(n=7)$

1. Intranasal $5 \times 10^{5}$ MSC day

7 and $9(n=9)$

2. Intranasal saline day 7 and $9(n=10)$

Intranasal $5 \times 10^{5}$ MSC day 7 and $9(n=12)$.

1. Intrastriatal saline $(n=9)$

2. Intrastriatal $1 \times 10^{5} \mathrm{hMSCs}$ $(n=8)$
Outcome $\left(^{*}=\mathrm{p}<0.05\right.$

$\left.*^{*}=p<0.01 / /^{* * *}=p<0.001\right)$

- Rotational behavior (turns/min)

1. No-intervention group: $23.8 \pm 2.1$

2. Control saline-treated group: $25.1 \pm 1.7$

3. MSC-treated group: $14.1 \pm 3.3^{*}$

4. Enriched rMSC-treated group: $10.8 \pm 1.7^{*}$

- TH-positive neurons:

1. No-intervention group: $24.2 \pm 6.7 \%$

4. Enriched riMSC-treated group: $52.5 \pm 8.2 \% *$

- Amphetamine-induced rotational behavior

1. Control group: $8.5 \pm 3.5$ turns/min

2. Fibroblast group: $8.2 \pm 3.3$ turns/min

3. rMSC group:

$1.2 \pm 0.7$ turns/min*

- Cylinder test

1. Control group:

$64.7 \pm 17.3 \%$

2. Fibroblast group:

$60.2 \pm 16.1 \%$

3. rMSC group:

$29.3 \pm 13.7 \%$ *

- Preservation of $\mathrm{TH}^{+}$cells: $3^{*}>2>1$

- Stepping ratio (contralateral/ ipsilateral) MSC-treated group $2(0.7)^{* *}>$ group $1(0.1)$

- Amphetamine-induced rotational behavior

MSC-treated group $4^{*}<$ group 3

- Histology

a. Group 4: $24 \%$ of MSCs survived in central nervous system for at least 4.5 months

b. $\mathrm{TH}^{+}$cell survival:

Group $2^{*}>1$ and $4^{*}>3$

c. Inflammatory cytokines Group $2^{*}<1$ and $4^{*}<3$

- Apomorphine-induced rotational behavior

1. No effect 


\begin{tabular}{|c|c|c|c|c|}
\hline & & & & 2. Reduced rotational behavior* \\
\hline & & & & $\begin{array}{l}\text { - Expression of GDNF increased } \\
\text { in hMSCs group }\end{array}$ \\
\hline & & & & $\begin{array}{l}\text { - Apoptosis decreased in } \\
\text { hMSCs treated group }\end{array}$ \\
\hline \multirow{3}{*}{$\begin{array}{l}\text { Cova, Armentero } \\
\text { et al. [57] }\end{array}$} & \multirow[t]{3}{*}{ hMSCs } & \multirow[t]{3}{*}{$5 / 28$ days } & SHAM unilateral lesion & \multirow{3}{*}{$\begin{array}{l}\text { - Dose-dependent neurorescue } \\
\text { effects (hMSCs vs saline) in } \\
\text { unilateral 6-OHDA lesioned, } \\
\text { but not SHAM lesioned, } \\
\text { rats with }\end{array}$} \\
\hline & & & 1.Intrastriatal saline & \\
\hline & & & $\begin{array}{l}\text { 2. Intrastriatal } 3.2 \times 10^{4} \text { hMSCs } \\
(n=6-10)\end{array}$ & \\
\hline \multirow[t]{5}{*}{ Male rats } & & & $\begin{array}{l}\text { 3. Intrastriatal } 1.8 \times 10^{5} \mathrm{hMSCs} \\
(\mathrm{n}=6-10)\end{array}$ & \multirow{2}{*}{$\begin{array}{l}\text { a) Reduction }{ }^{* * *} \text { ongoing } \\
\text { toxin-induced degeneration } \\
\text { of dopaminergic terminals }\end{array}$} \\
\hline & & & 6-OHDA unilateral lesion & \\
\hline & & & 1. Intrastriatal saline & \multirow{2}{*}{$\begin{array}{l}\text { b) Enhanced neurogenesis*/** } \\
\text { (neural progenitor cells) in the } \\
\text { periventricular zone }\end{array}$} \\
\hline & & & $\begin{array}{l}\text { 2. Intrastriatal } 3.2 \times 10^{4} \text { hMSCs } \\
(n=6-10)\end{array}$ & \\
\hline & & & $\begin{array}{l}\text { 3. Intrastriatal } 1.8 \times 10^{5} \mathrm{hMSCs} \\
(\mathrm{n}=6-10)\end{array}$ & $\begin{array}{l}\text { c) Persistent release of specific } \\
\text { cytokines }\end{array}$ \\
\hline \multirow{3}{*}{$\begin{array}{l}\text { Delcroix, Garbayo } \\
\text { et al. [37] }\end{array}$} & \multirow[t]{3}{*}{ rMSCs } & \multirow[t]{3}{*}{$14 / 64$ days } & 1. Intrastriatal saline $(n=6)$ & - Rotational behavior (turns/min): \\
\hline & & & $\begin{array}{l}\text { 2. Intrastriatal } 1.5 \times 10^{5} \mathrm{rMSCs} \\
(\mathrm{n}=6)\end{array}$ & 1. saline treated group: 18.5 \\
\hline & & & $\begin{array}{l}\text { 3. Intrastriatal } 1.5 \times 10^{5} \\
\text { riMSCs }+P(n=6)\end{array}$ & 2. rMSCs-treated group: 17.5 \\
\hline \multirow[t]{3}{*}{ Female rats } & \multirow[t]{2}{*}{ riMSCs } & & \multirow{2}{*}{$\begin{array}{l}\text { 4. Intrastriatal } 1.5 \times 10^{5} \\
\text { riMSCs }+P+N T 3(n=6)\end{array}$} & 3. riMSCs + $P$ treated group: $8.5^{*}$ \\
\hline & & & & $\begin{array}{l}\text { 4. riMSCs + P + NT3 treated } \\
\text { group: } 3.0^{*}\end{array}$ \\
\hline & riMSCs + P & & & $\begin{array}{l}\text { - Preservation of } \mathrm{TH}^{+} \text {cells : } \\
4^{*}>3>2>1\end{array}$ \\
\hline \multirow[t]{4}{*}{$\begin{array}{l}\text { Levy, Bahat-Stroomza } \\
\text { et al. [56] Male rats }\end{array}$} & hMSCs & $35 / 125$ days & $\begin{array}{l}\text { 1) Intrastriatal saline in } 5 \\
(n=7)\end{array}$ & $\begin{array}{l}\text { - Rotational behavior (turns/min) } \\
\text { (post-lesional 100\%) }\end{array}$ \\
\hline & \multirow[t]{3}{*}{ hiMSCs (neural phenotype) } & & $\begin{array}{l}\text { 2) Intrastriat. } 5 \times 10^{5} \mathrm{MSC}^{\prime} \mathrm{s} \\
(\mathrm{n}=7)\end{array}$ & 1) saline-treated group: $88 \%$ \\
\hline & & & \multirow{2}{*}{$\begin{array}{l}\text { 3) Intrastriat. } 5 \times 10^{5} \text { neural } \\
\text { iMSC's }(n=7)\end{array}$} & 2) hMSCs-treated group: 90\% \\
\hline & & & & 3) hiMSCs-treated group: 42\%* \\
\hline $\begin{array}{l}\text { Sadan, Bahat-Stromza } \\
\text { et al. [55] }\end{array}$ & hMSCs & $1 \mathrm{hr} / 42$ days & 1) Intrastriatal saline $(n=10)$ & $\begin{array}{l}\text { - D-amphetamine-induced } \\
\text { rotational behavior }\end{array}$ \\
\hline \multirow[t]{6}{*}{ Male rats } & \multirow[t]{6}{*}{ hiMSCs (BDNF/GDNF) } & & $\begin{array}{l}\text { 2) Intrastriatal } 1.5 \text { or } \\
4.5 \times 10^{5} \text { hMSCs }(n=21)\end{array}$ & $\begin{array}{l}\text { 1. saline group: increase } \\
4.74 \pm 1.07 \text { turns/min }\end{array}$ \\
\hline & & & & $\begin{array}{l}\text { 2. MSCs group: increase } \\
2.86 \pm 0.54 \text { turns/min }\end{array}$ \\
\hline & & & $\begin{array}{l}\text { 3. Intrastriatal } 1.5 \text { or } \\
4.5 \times 10^{5} \text { hiMSCs }(n=21)\end{array}$ & $\begin{array}{l}\text { 3. iMSCs group: increase } \\
2.16 \pm 0.37^{*} \text { turns } / \mathrm{min}\end{array}$ \\
\hline & & & & $\begin{array}{l}\text { - TH-positive area (treated } \\
\text { versus untreated site) }\end{array}$ \\
\hline & & & & $\begin{array}{l}\text { 2. hMSCs group: treated site > } \\
\text { untreated site }\end{array}$ \\
\hline & & & & $\begin{array}{l}\text { 3. hiMSCs group: treated } \\
\text { site }^{*}>\text { untreated site }\end{array}$ \\
\hline \multirow{3}{*}{$\begin{array}{l}\text { Zhu, Ma et al. [59] } \\
\text { Male rats }\end{array}$} & \multirow[t]{3}{*}{ rNSCs } & \multirow[t]{3}{*}{$35 / 155$ days } & 1. No intervention $(n=13)$ & - Rotational behavior: \\
\hline & & & $\begin{array}{l}\text { 2. Intranigral(SNc) } 5 \times 10^{4} \\
\text { rNSCs }(n=20)\end{array}$ & $\begin{array}{l}\text { 1. Group without intervention: } \\
233.9 \pm 70.43\end{array}$ \\
\hline & & & $\begin{array}{l}\text { 3. Intrastriatal } 5 \times 10^{4} \mathrm{rNSCs} \\
(\mathrm{n}=5)\end{array}$ & $\begin{array}{l}\text { 2. rNSCs SNc group: } \\
189.3 \pm 63.24^{* * *}\end{array}$ \\
\hline
\end{tabular}




\section{Table 1 Placebo-controlled stem cell applications in rodent animal models of parkinsonism (Continued)}

\begin{tabular}{|c|c|c|c|c|}
\hline & & & & $\begin{array}{l}\text { 3. rNSCs Intrastriatal group: } \\
169.3 \pm 47.28^{*}\end{array}$ \\
\hline & & & & $\begin{array}{l}\text { - TH-positive cells in the } \\
\text { SNc: } 2>1\end{array}$ \\
\hline & & & & $\begin{array}{l}\text { - EGFP-labeled NSCs } \\
\text { identified as TH+ } \text { THlls }^{+} \\
\text {in } 2 \text { and } 3\end{array}$ \\
\hline \multirow[t]{9}{*}{$\begin{array}{l}\text { Ramos-Moreno, Castillo } \\
\text { et al. [61] Female rats }\end{array}$} & hNSCs & 45/165 days & 1. Intrastriatal saline $(n=15)$ & $\begin{array}{l}\text { - D-amphetamine-induced } \\
\text { rotational behavior: }\end{array}$ \\
\hline & hiNSCs & & $\begin{array}{l}\text { 2. Intrastriatal } 3 \times 10^{5} \text { hNSCs } \\
(n=17)\end{array}$ & 1. Control group: 18 turns/min \\
\hline & (expressing $\mathrm{BCl}-\mathrm{X}_{\mathrm{L}}$ ) & & $\begin{array}{l}\text { 3. Intrastriatal } 3 \times 10^{5} \text { hiNSCs } \\
\mathrm{BCl}-\mathrm{X}_{\mathrm{L}} \text { expression }(\mathrm{n}=21)\end{array}$ & $\begin{array}{l}\text { 2. hNSCs-treated group: } \\
17 \text { turns/min }\end{array}$ \\
\hline & & & & $\begin{array}{l}\text { 3. hiNSCs-treated group: } \\
3 \text { turns/min*** }\end{array}$ \\
\hline & & & & $\begin{array}{l}\text { - Apomorphine-induced } \\
\text { rotational behavior: }\end{array}$ \\
\hline & & & & 1. Control group: 6.5 turns/min \\
\hline & & & & $\begin{array}{l}\text { 2. hNSCs-treated group: } \\
2 \text { turns/min** }\end{array}$ \\
\hline & & & & $\begin{array}{l}\text { 3. hiNSCs-treated group: } \\
\text { 2.5/min** }\end{array}$ \\
\hline & & & & $\begin{array}{l}\text { - Paw mobility test: } \\
3^{* *}>2^{*}>1\end{array}$ \\
\hline
\end{tabular}

Abbreviations: 6-OHDA 6 hydroxydopamine, ASCs Adult stem cells, MSCs Mesenchymal stem cells, NSCs Neural stem cells, $h$ human $r$ rat, $i$ induced or transduced, EGFP Enhanced Green Fluorescent Protein, BDNF Brain-Derived Neurotrophic Factor, GDNF Glial cell Derived Neurotrophic Factor, NT-3 Neurotrophine-3, $P$ Pharmacologically active microcarriers, $B C l-X_{L}$ anti-apoptotic granulocyte-colony stimulating factor enhancing the expression of key genes involved in dopaminergic patterning, differentiation and maturation); SNC Substantia Nigra pars compacta, $\mathrm{TH}^{+}$Tyrosine Hydroxylase Immunoreactive positive cells.

behavior showed a significant reduction of turns/minute. But for $4 / 7$ studies $[32,51,53,58]$, in all studies with expanded MSCs $[32,51,53,58]$, as well in all studies with expanded and enriched or epigenetically induced MSCs $[37,51,55,56]$, and expanded neural stem cells $[59,61]$, this reduction was established.

The administration of expanded, not-induced stem cells in these studies varied widely in relation to the onset of the toxin-induced motor parkinsonism. Stem cells were applied within 2 hours $[32,55]$, within 5 to 14 days $[57,58]$, or 30 days after the lesion [54,56,59-61]. Overlooking the effects in these studies on rotational behavior, the period in between application and lesioning seems to influence the clinical outcome: the earlier the application, the better the resulting clinical effects. Remarkably, after intravasal administration of ASCs, whether applied in the acute phase [32] or 3 weeks after lesioning [32], only a few ASCs (about $2 \%$ ) could be detected in the nigral substance, the majority was trapped in the lungs. Nevertheless, also in these experiments, a significant beneficial effect on motor behavior could be established [32]. Here, it seems relevant to mention that after intrastriatal (intracaudate) application in the non-human primate MPTP model, $1 \%$ of the implanted stem cells could be identified at the injection site, whereas over $80 \%$ was found to be migrated to and along the impaired nigrostriatal pathways [60].

As for the histological/histochemical findings, after intracerebral stem cell application, in all experiments, more striatal $\mathrm{TH}$-positive neurons were seen in the treated as compared to the non-treated, control lesioned animals, suggesting ASC-induced increased neuronal plasticity (neurorescue) with increased modulation of cell survival (and an increased striatal dopamine level), enhanced neurogenesis (progenitor cells in the subventricular zone), and a decreased modulation of inflammation, gliosis and death-signaling $[51,52,55,57,59,62]$. The same preservation of $\mathrm{TH}$-positive cells was also observed after intravenous [32] and intranasal MSC application [53].

\section{Clinical experience with ASC in motor parkinsonism}

As of yet, only three studies were reported, dealing with the effects of, intracerebral or intravasal applied, allogenic or autologous adult stem cells in patients suffering motor parkinsonism in $\mathrm{PD}$, multiple system atrophy (MSA) or progressive supranuclear palsy (PSP) [63-65]. The results are summarized in Table 3 . In most but not all PD patients, the subventricular application of both, allogenic and autologous bone marrow-derived mesenchymal stem cells did improve clinical scores of motor behavior, as expressed in a significant decrease of 
Table 2 Placebo-controlled stem cell applications in animal models of parkinsonism

\begin{tabular}{|c|c|c|c|c|}
\hline \multicolumn{5}{|c|}{ Striatal MPTP and subcutaneous proteasome inhibitor (MG-132) lesioned animals } \\
\hline $\begin{array}{l}\text { Authors } \\
\text { animals }\end{array}$ & $\begin{array}{l}\text { Product expanded } \\
\text { allogenic ASCs } \\
\text { Cyclosporin A }\end{array}$ & Applic/Observ time & Characteristics/Dosages/Application & $\begin{array}{l}\text { Outcome }\left(^{*}=p<0.05 /\right. \\
* *=p<0.01 / * * *=p<0.001)\end{array}$ \\
\hline \multirow[t]{2}{*}{ Chao, He et al. [54] } & \multirow[t]{2}{*}{ mMSCs } & \multirow{3}{*}{$\begin{array}{l}\text { Directly after last MPTP } \\
\text { injection/1 month }\end{array}$} & 1. Intraperitoneal saline $(n=24)$ & . $\mathrm{SN} \mathrm{TH}^{+}$cells: $3^{* *}>2$ \\
\hline & & & $\begin{array}{l}\text { 2. Intraperitoneal MPTP }+ \text { IV saline } \\
(n=24)\end{array}$ & - SN microglial cells: $3^{*}<2$ \\
\hline Male C57BL/6 mice & & & $\begin{array}{l}\text { 3. Intraperitoneal MPTP }+ \text { IV } 10^{5} \text { mMSCs } \\
(\mathrm{n}=24)\end{array}$ & $\begin{array}{l}\text { Phagocytosis and Complement } \\
\text { inhibition: } 3^{*}<2\end{array}$ \\
\hline \multirow[t]{2}{*}{ Park, Bang et al. [62] } & \multirow[t]{6}{*}{ hiMSCs } & \multirow[t]{6}{*}{$\begin{array}{l}1 \text { day after last MPTP and } \\
\text { 3-NP injection/28 days }\end{array}$} & 1. Intraperitoneal saline-treated $(n=10)$ & $\begin{array}{l}\text { - Group } 2 \text { and 3: } 48 \% \text { loss of } \\
\text { nigral cells; }\end{array}$ \\
\hline & & & 2. Intraperitoneal MPTP + 3-NP $(n=8)$ & - Group 3 compared to group 2: \\
\hline \multirow[t]{4}{*}{ Male C57BL/6 mice } & & & \multirow[t]{4}{*}{$\begin{array}{l}\text { 3. Intraperitoneal MPTP }+3-\mathrm{NP} \text { and } \\
\text { IV } 1 \times 10^{6} \text { hiMSCs in } 200 \mu \mathrm{I}(\mathrm{n}=8)\end{array}$} & $\begin{array}{l}\text { a. } 2 \% \text { of hiMSCs in } \mathrm{SN} \text {, and } \\
4 \% \text { in the Striatum }\end{array}$ \\
\hline & & & & $\begin{array}{l}\text { b. Motor behavior improved* } \\
\text { during } 10 \text { days }\end{array}$ \\
\hline & & & & $\begin{array}{l}\text { c. Increased modulation of } \\
\text { cell survival* and decreased } \\
\text { modulation of death-signaling } \\
\text { pathways, with } 20 \% \text { cell survival** }\end{array}$ \\
\hline & & & & $\begin{array}{l}\text { d. Decreased modulation of } \\
\text { inflammation** and gliosis**, } \\
\text { with a marked decrease of } \\
\text { activated microglia** and } \\
\text { astrocytes }^{* * *}\end{array}$ \\
\hline $\begin{array}{l}\text { Bjugstad, Teng } \\
\text { et al. [60] }\end{array}$ & \multirow[t]{2}{*}{ hiNSCs } & \multirow[t]{2}{*}{$\begin{array}{l}4 \text { and/or } 6 \text { months after last } \\
\text { MPTP injection/4 }(n=3) \\
\text { and } 7 \text { months }(n=4)\end{array}$} & \multirow{2}{*}{$\begin{array}{l}\text { Bilateral intrastriatal and unilateral } \\
\text { intranigral implantation of } \\
\text { each } 10^{6} \text { hiNSCs }(n=7) \text { in the } \\
\text { intramuscular MPTP lesioned } \\
\text { monkey }\end{array}$} & $\begin{array}{l}\cdot>80 \% \text { of hiNSCs immigrated } \\
\text { along the impaired nigrostriatal } \\
\text { pathway }\end{array}$ \\
\hline $\begin{array}{l}\text { African green } \\
\text { Monkeys }\end{array}$ & & & & $\begin{array}{l}\cdot<1 \% \text { of a total of } 2 \times 10^{6} \text { hiNSCs } \\
\text { implanted within the caudate } \\
\text { nucleus (intrastriatal) was } \\
\text { identified at this site. }\end{array}$ \\
\hline \multirow[t]{3}{*}{ Park, Lee et al. [52] } & \multirow[t]{3}{*}{ hMSCs } & \multirow[t]{3}{*}{$\begin{array}{l}21 \text { days } / 3,4,6,7 \\
10 \text { weeks }\end{array}$} & 1. MG-132 lesioned rats & $\begin{array}{l}\cdot 1.7 \% \text { hMSCs detected in the } \\
\text { nigral substance }\end{array}$ \\
\hline & & & \multirow{2}{*}{$\begin{array}{l}\text { 2. MG-132 lesioned rats treated } \\
\text { during } 3 \text { weeks with weekly } \\
\text { intravasal application of } 10^{6} \text { hMSCs }\end{array}$} & $\begin{array}{l}\text { - Survival of nigral and striatal } \\
\mathrm{TH}^{+} \text {cells* after hMSCs }\end{array}$ \\
\hline & & & & $\begin{array}{l}\text { - Increased striatal dopamine } \\
\text { level* after hMSCs }\end{array}$ \\
\hline Male rats & & & & $\begin{array}{l}\text { - Reduction* in microglia } \\
\text { activation after hMSCs }\end{array}$ \\
\hline
\end{tabular}

Abbreviations: ASCs Adult stem cells, IV Intravenous, MPTP 1-methyl-4-phenyl-1,2,3,6-tetrahydropyridine, MSCs Mesenchymal stem cells, NSCs Neural stem cells, $h$ human $m$ mouse, $i$ induced or transduced, MG-132 carbobenzoxy-Leu-Leu-leucinal, a proteasome inhibitor. SN Substantia Nigra, $T H^{+}$Tyrosine Hydroxylase Immunoreactive positive cells.

UPDRS scores in ON and OFF conditions. Interestingly, these effects did appear faster and more outspoken the earlier these interventions were applied, mimicking the results in preclinical experiments. Also Magnetic Resonancetractography of degenerated dopaminergic projections did show significant improvements in those patients.

In MSA and PSP patients, compared to placebotreated patients, in some patients, a temporary improvement [60] or reduced deterioration in motor and cognitive functions [60] witnessed a reduction in natural progressive deterioration with subventricular or intravasal ASC implantations. This reduced disease progression in these patients was also found reflected in MRI (Magnetic Resonance Imaging)- and FDG PET (fluorodeoxyglucose positron emission tomography)imaging, showing less atrophy and less decreased glucose metabolism in cortex and cerebellum.

\section{Discussion}

PD is a chronic progressive, diffuse $\alpha$-synucleinopathic disease of the central nervous system in which (symptomatic) therapeutic strategies aim to compensate for the striatal dopamine deficiency in order to mainly decrease motor symptomatology. As of yet, protective/ repairing therapeutic strategies in $\mathrm{PD}$ are an unmet need. 
Table 3 Open label and placebo-controlled stem cell applications in clinical parkinsonism

\begin{tabular}{|c|c|c|c|c|}
\hline Authors Patients & Product & $\begin{array}{l}\text { Observ. time } \\
\text { (months) }\end{array}$ & Characteristics/Dosages/Application & Outcome $\left(^{*}=p<0.05 / * *=p<0.01\right)$ \\
\hline $\begin{array}{l}\text { Venkataramana, } \\
\text { Pal et al. [65] }\end{array}$ & Allogenic & $3,6,12$ & $\begin{array}{l}\text { 1. PD patients with bilateral subventricular } \\
\text { intracerebral application of } 2 \times 10^{6} / \mathrm{kg} \\
\text { bodyweight MSCs in } 2 \mathrm{ml}(\mathrm{n}=8)\end{array}$ & UPDRS in ON/OFF: \\
\hline PD patients & MSCs & & $\begin{array}{l}\text { 2. MSA + PSP patients with bilateral } \\
\text { subventricular intracerebral application } \\
\text { of } 2 \times 10^{6} / \mathrm{kg} \text { bodyweight MSCs in } \\
2 \mathrm{ml}(\mathrm{n}=4)\end{array}$ & $\begin{array}{l}\text { a. In PD patients: permanently improved* } \\
\text { compared with baseline during both ON } \\
\text { (18\%: } 51.2 \text { versus } 62.3 \text { ) and OFF ( } 31.2 \% \text { : } \\
59.5 \text { versus } 86.5 \text { ). Effect stronger in patients } \\
\text { with disease duration }<5 \text { years (ON } 45.5 \% \text { / } \\
\text { OFF } 56.7 \% \text { ) as compared to patients with a } \\
\text { duration > } 10 \text { years (ON 6.3\% OFF 12.4\%). }\end{array}$ \\
\hline MSA patients & & & & $\begin{array}{l}\text { b. Some MSA/PSP patients temporarily } \\
\text { improved. The effect was not correlated } \\
\text { with disease severity and disease } \\
\text { duration.MR-tractography: }\end{array}$ \\
\hline PSP patients & & & & $\begin{array}{l}\text { a. PD patients, after implantation, did show } \\
\text { a trend of steadily improvement in } \\
\text { tractographical images in genu and } \\
\text { peduncles. }\end{array}$ \\
\hline Open Label & & & & $\begin{array}{l}\text { b. MSA/PSP patients showed further } \\
\text { reduction of tractographical images after } \\
\text { stem cell implantation. }\end{array}$ \\
\hline $\begin{array}{l}\text { Venkataramana, } \\
\text { Kumar et al. [64] }\end{array}$ & $\begin{array}{l}\text { Autologous } \\
\text { MSCs }\end{array}$ & $10-36$ & $\begin{array}{l}\text { PD patients }(n=7) \text { with an UPDRS ON/OFF } \\
\text { score } 50.6 / 65.0 \text { and a mean disease duration }\end{array}$ & UPDRS in ON/OFF: \\
\hline PD patients & & & $\begin{array}{l}\text { of } 14.7 \mathrm{yr} \text { treated with } 10^{\circ} \mathrm{MSCs} / \mathrm{kg} \\
\text { bodyweight in the subventricular zone }\end{array}$ & $\begin{array}{l}\text { a. In } 3 / 7 \text { patients there was a stable } \\
\text { improvement of ON/OFF scores of } \\
38 \% \text { respect } 22.9 \% \text { with unchanged } \\
\text { anti-parkinsonian medication. }\end{array}$ \\
\hline \multirow[t]{2}{*}{ Open Label } & & & & $\begin{array}{l}\text { b. In } 3 / 7 \text { patients after treatment, only } \\
\text { marginal clinical effects were observed }\end{array}$ \\
\hline & & & & $\begin{array}{l}\text { Anti-parkinsonian medication significantly } \\
\text { reduced in } 2 \text { patients. }\end{array}$ \\
\hline \multirow[t]{2}{*}{ Lee, kim et al. [63] } & \multirow{2}{*}{$\begin{array}{l}\text { Autologous } \\
\text { MSCs }\end{array}$} & \multirow{2}{*}{$\begin{array}{l}1,2,3,4,5,6,8 \\
10,12\end{array}$} & \multirow{2}{*}{$\begin{array}{l}\text { Patients with Cognitive intact MSA-C } \\
\text { (with UMSARS scores between } 30 \text { to 50) }\end{array}$} & UMSARS score \\
\hline & & & & $\begin{array}{l}\text { a. MSCs-treated patients showed a } \\
\text { reduced* increase of UMSARS score } \\
\text { compared to placebo treated patients. }\end{array}$ \\
\hline \multirow[t]{3}{*}{ MSA patients } & & & \multirow[t]{3}{*}{$\begin{array}{l}\text { 1. Placebo group: Intravenous or intra-arterial } \\
\text { placebo }(n=17)\end{array}$} & $\begin{array}{l}\text { b. Intra-arterial application of MSCs was } \\
\text { complicated with some MRI-detectable } \\
\text { ischemic lesions }\end{array}$ \\
\hline & & & & Cognitive functions: \\
\hline & & & & $\begin{array}{l}\text { Significantly* worsened in the placebo, } \\
\text { but not in the MSCs-treated patients }\end{array}$ \\
\hline \multirow{2}{*}{$\begin{array}{l}\text { Placebo } \\
\text { controlled }\end{array}$} & & & \multirow{2}{*}{$\begin{array}{l}\text { 2. MSCs group: } 4 \times 10^{6} \text { MSCs intravenously } \\
\text { or intra-arterial }(n=14)\end{array}$} & MRI and FDG PET: \\
\hline & & & & $\begin{array}{l}\text { Showed significantly increased* gray cerebral } \\
\text { cortical areas respectively more decreased } \\
\text { cortical and cerebellar glucose metabolism } \\
\text { in placebo-treated, as compared to } \\
\text { MSCs-treated patients. }\end{array}$ \\
\hline
\end{tabular}

Abbreviations: MSCS Mesenchymal stem cells, MSA multiple system atrophy, MSA-C multiple system atrophy, cerebellar type, PSP progressive supranuclear palsy MRI magnetic resonance imaging, UMSARS unified MSA rating scale, FDG PET fluorodeoxyglucose positron emission tomography.

Generally speaking, stem cells, and specifically adult stem cells, pending their environment after re-implantation or pending their in-vitro induction, are supposed to not only differentiate into functional (neuronal) cells, including dopamine producing neurons, but to also easily expand and thus to deliver enough cells for transplantation.
Although differentiation and proliferation of bone marrowderived stem cells are jeopardized by aging and chronic diseases including diabetes [66], renal failure [67] and ALS (Amyotrophic lateral sclerosis) [68], in PD patients, up to the $15^{\text {th }}$ passage, ASCs are fully comparable to those of healthy age-matched individuals regarding phenotype, 
morphology and capacity to multi-differentiate [69], and are also able to inhibit $\mathrm{T}$ lymphocyte proliferation induced by mitogens.

Next to the multi-differentiated proliferative capacity, adult stem cells, and especially expanded and induced ASC's, may secrete an array of trophic factors. As a matter of fact they may specialize to express mainly such factors by induction in vitro through exposure to trophic factors (including epidermic growth factor EGF and basic fibroblast growth factor bFGF), by transduction with a viral vector and/or by binding these ASCs to pharmacologically active microcarriers, containing trophic factors such as NT-3 prior to the application. When adding epidermal growth factor (EGF) and basic fibroblast growth factor (bFGF) to serum-free medium, in order to expand and induce human ASCs, the secretion of neurotrophic factors such as BDNF (normally neglectable) will increase to $125 \pm 12 \mathrm{pg} /$ day $/ 10^{6}$ ASCs [70]. Thus induced ASCs will function as a vehicle for adequate delivery of neurotrophic factors (BDNF and GDNF) when transplanted in the central nervous system $[55,70]$.

It is suggested that ASC-produced neurotrophic factors such as GDNF and BDNF enhance neuroprotection with an increased modulation of cell survival and a decreased modulation of inflammation, gliosis and death signaling. These trophic factors thus stimulate neurorescue by better protection of $\alpha$-synucleinopathic jeopardized neurons, including DA producing neurons. This effect was also reported in a MPTP animal model in which allogenic neural stem cells were applied unilateral [71]. The jeopardized DA producing neurons were rescued and this effect was also observed in the opposite side of the $\mathrm{SN}$ in which no stem cells were applied [71]. So, in PD patients, autologous ASCs, and especially expanded and/or induced ASCs, due to their capacity to increase neuroplasticity, theoretically not only offer an increased neuroprotection, but also an increased neuro-repair in PD patients resulting in a slowing down of natural progression in this debilitating disease.

In preclinical studies, the stimulated secretion of GDNF and BDNF by ASCs is found to result in a higher number of striatal $\mathrm{TH}$-positive neurons in the rat lesioned striatum as compared to placebo-treated rats, accompanied with a higher striatal dopamine level and decreased rotational behavior [58,70]. Other ASCproduced trophic factors, such as basic fibroblast growth factor bFGF and epidermal growth factor EGF, seem to be more relevant for neurorestoration (synthesis of extracellular matrix). In rodents, the intranigral application of (adenovirus mediated) glial cell line derived neurotrophic factor (GDNF), one week before the ipsilateral nigral 6OHDA lesioning, did rescue $70 \%$ of the nigral TH-positive cells as compared to $30 \%$ in the not-pretreated, lesioned (control) animals, resulting in a significantly reduced rotational behavior: $5.4 \pm 15.2$ turns/15-minutes versus $40.8 \pm 25$ turns $/ 15$-minutes $(\mathrm{p}<0.05)$ [72]. Note however that each intracerebral needle-induced manipulation per se might initiate the secretion of the same neurotrophic factors and/or cytokines mimicking a clinical effect, which may confound the results of a placebo treatment $[73,74]$.

In the preclinical studies, reviewed above, two studies were performed in which ASCs were locally applied within 2 hours after the application of a 6-OHDA unilateral lesion in rodents $[32,55]$. Both studies, reported a significant improvement in rotational behavior and in survival of $\mathrm{TH}$ positive neurons within 4-6 weeks. In the studies applying ASCs, and especially expanded noninduced ASCs, later on, in a more chronic phase of a lesion, no significant reduction in rotational behavior could be established [28]. In translation, the clinical effects of implantation of expanded MSCs in PD patients might profit their application in an early phase of their disease. Indeed, in PD patients, the clinical effects of stem cell application seem to relate with disease duration [60].

Finally, as ASCs are large cells, unable to cross the blood-brain barrier [44], intracerebral application seems to be superior to intravasal application, as only $1-2 \%$ of thus applied cells will reach the central nervous system [52]. Intranasal administration of MSCs, however, might offer an alternative, as this way of application in unilateral 6-OHDA-lesioned rodents did result in both, a significantly improved motor behavior and a significant decrease in inflammatory cytokines (suggesting a strong cell protective effect by inhibiting inflammation cascades) with $24 \%$ of the cells surviving for at least 4.5 month in the central nervous system [53].

\section{Conclusions}

In conclusion

- ASCs are capable of migrating to the lesioned cells/ organs throughout the body, a phenomenon, which is called homing.

- ASCs are easily to harvest out of the iliac crest. Their number might be increased by expanding them, and their functions might be developed prior to re-application by inducing them. Unlike ASCs in patients with other chronic diseases, in PD patients those cells are not impaired.

- Expanded and/or induced ASCs may stabilize motor (and non-motor) parkinsonism in patients suffering PD and may be also Parkinson-plus syndromes, by increasing neural plasticity rather than by differentiating into neurons. Early in the disease, ASCs will promote neurorescue/neuroprotection by increasing immune modulation and reducing inflammation), especially when expanded 
(undifferentiated) ASCs are administered. Later in the disease, they mainly will promote neurorestoration, especially when induced (differentiated) ASCs are given.

- Neurotrophic factors such as BDNF and GDNF (mainly interfering with destructive pathways) play a major role in neuroprotection, whereas other growth factors such as EGF and bFGF (promoting synthesis of extracellular matrix) are more important in neurorepair. The best time to start with ASCs administration is in the very early phase of Parkinson's disease.

\section{Competing interests}

The authors declare that they have no competing interests.

\section{Authors' contributions}

$\mathrm{JdM}$ compiled the article and did the literature search and summarized the preclinical and clinical studies as a part of his PhD. JDM wrote the draft and invited the 2 co-authors to comment and revised the draft. EW is the promoter of JdM and revised the article critically and was also responsible for the completeness of the issues addressed in this article. $\mathrm{CL}$ as an experienced neurologist familiar with Parkinsonism revised the article to assure the translational aspects of the content and was especially responsible for revising the clinical part of the article. All authors read and approved the final manuscript.

\section{Author details}

${ }^{1}$ Department of Neurosciences University Maastricht, Maastricht, The Netherlands. ${ }^{2}$ Amarna Stem Cells Group, Maastricht, The Netherlands. ${ }^{3}$ Department of Neurology, Asan Medical Center University of Ulsan, Seoel, South Korea. ${ }^{4}$ Department of Neurology, UniversitatsSpital, Zurich, Switzerland.

\section{Received: 29 January 2013 Accepted: 2 June 2013}

Published: 4 June 2013

\section{References}

1. Wirdefeldt K, Adami HO, Cole P, Trichopoulos D, Mandel J: Epidemiology and etiology of Parkinson's disease: a review of the evidence. Eur $J$ Epidemiol 2011, 26(Suppl 1):S1-S58.

2. Dorsey ER, Constantinescu R, Thompson JP, Biglan KM, Holloway RG, Kieburtz $K$, et al: Projected number of people with Parkinson disease in the most populous nations, 2005 through 2030. Neurology 2007, 68(5):384-386.

3. Diem-Zangerl A, Seppi K, Oberaigner W, Poewe W: Mortality in Parkinson's disease, a 20-year follow-up study. Mov Disord 2010, 25(5):661-662.

4. Driver JA, Kurth T, Buring JE, Gaziano JM, Logroscino G: Parkinson disease and risk of mortality: a prospective comorbidity-matched cohort study. Neurology 2008, 70(16 Pt 2):1423-1430.

5. Wolters EC, Van Laar T, Berendse HW: Parkinsonism and related disorders. Third Editionth edition. VU University Press; 2010:143-158.

6. Marsden CD: Movement Disorders. Oxford Textbook of Medicine 1996, 3:3998-4022.

7. Fox SH, Katzenschlager R, Lim SY, Ravina B, Seppi K, Coelho M, et al: The Movement Disorder Society Evidence-Based Medicine Review Update: Treatments for the motor symptoms of Parkinson's disease. Mov Disord 2011, 26(Suppl 3):S2-S41.

8. Fillmore HL, Holloway KL, Gillies GT: Cell replacement efforts to repair neuronal injury: a potential paradigm for the treatment of Parkinson's disease. NeuroRehabilitation 2005, 20(3):233-242.

9. Hall VJ, Li JY, Brundin P: Restorative cell therapy for Parkinson's disease: a quest for the perfect cell. Semin Cell Dev Biol 2007, 18(6):859-869.

10. Laguna Goya R, Tyers P, Barker RA: The search for a curative cell therapy in Parkinson's disease. J Neurol Sci 2008, 265(1-2):32-42.

11. Meyer AK, Maisel M, Hermann A, Stirl K, Storch A: Restorative approaches in Parkinson's Disease: which cell type wins the race? J Neurol Sci 2010, 289(1-2):93-103.

12. Parish $\mathrm{CL}$, Arenas $\mathrm{E}$ : Stem-cell-based strategies for the treatment of Parkinson's disease. Neurodegener Dis 2007, 4(4):339-347.
13. Fujikawa T, Oh SH, Pi L, Hatch HM, Shupe T, Petersen BE: Teratoma formation leads to failure of treatment for type I diabetes using embryonic stem cell-derived insulin-producing cells. Am J Pathol 2005, 166(6):1781-1791.

14. Hentze $H$, Graichen R, Colman A: Cell therapy and the safety of embryonic stem cell-derived grafts. Trends Biotechnol 2007, 25(1):24-32.

15. Lee AS, Tang C, Cao F, Xie X, van der Bogt K, Hwang A, et al: Effects of cell number on teratoma formation by human embryonic stem cells. Cell Cycle 2009, 8(16):2608-2612.

16. Ben-David U, Benvenisty N, Mayshar Y: Genetic instability in human induced pluripotent stem cells: classification of causes and possible safeguards. Cell Cycle 2010, 9(23):4603-4604.

17. Sadan O, Shemesh N, Cohen Y, Melamed E, Offen D: Adult neurotrophic factor-secreting stem cells: a potential novel therapy for neurodegenerative diseases. Isr Med Assoc J 2009, 11(4):201-204

18. de Munter JP, Wolters EC: Autologous stem cells in neurology: is there a future? J Neural Transm 2013, 120(1):65-73.

19. Aggarwal S, Pittenger MF: Human mesenchymal stem cells modulate allogeneic immune cell responses. Blood 2005, 105(4):1815-1822.

20. Zappia E, Casazza S, Pedemonte E, Benvenuto F, Bonanni I, Gerdoni E, et al: Mesenchymal stem cells ameliorate experimental autoimmune encephalomyelitis inducing T-cell anergy. Blood 2005, 106(5):1755-1761.

21. Barzilay R, Kan I, Ben-Zur T, Bulvik S, Melamed E, Offen D: Induction of human mesenchymal stem cells into dopamine-producing cells with different differentiation protocols. Stem Cells Dev 2008, 17(3):547-554.

22. Tropel P, Platet N, Platel JC, Noel D, Albrieux M, Benabid AL, et al: Functional neuronal differentiation of bone marrow-derived mesenchymal stem cells. Stem Cells 2006, 24(12):2868-2876.

23. Ye Y, Zeng YM, Wan MR, Lu XF: Induction of human bone marrow mesenchymal stem cells differentiation into neural-like cells using cerebrospinal fluid. Cell Biochem Biophys 2011, 59(3):179-184.

24. Zeng R, Wang LW, Hu ZB, Guo WT, Wei JS, Lin H, et al: Differentiation of Human Bone Marrow Mesenchymal Stem Cells into Neuron-like Cells in Vitro. Spine 2011, 36(13):997-1005.

25. Gnecchi M, Zhang Z, Ni A, Dzau VJ: Paracrine mechanisms in adult stem cell signaling and therapy. Circ Res 2008, 103(11):1204-1219.

26. Xu YX, Chen L, Wang R, Hou WK, Lin P, Sun L, et al: Mesenchymal stem cell therapy for diabetes through paracrine mechanisms. Med Hypotheses 2008, 71(3):390-393.

27. Jager M, Hernigou P, Zilkens C, Herten M, Li X, Fischer J, et al: Cell therapy in bone healing disorders. Orthopedic reviews 2010, 2(2):79-87.

28. Croft AP, Przyborski SA: Mesenchymal stem cells expressing neural antigens instruct a neurogenic cell fate on neural stem cells. Exp Neurol 2009, 216(2):329-341.

29. Tondreau T, Lagneaux L, Dejeneffe M, Massy M, Mortier C, Delforge A, et al: Bone marrow-derived mesenchymal stem cells already express specific neural proteins before any differentiation. Differentiation 2004, 72(7):319-326.

30. Wilkins A, Kemp K, Ginty M, Hares K, Mallam E, Scolding N: Human bone marrow-derived mesenchymal stem cells secrete brain-derived neurotrophic factor which promotes neuronal survival in vitro. Stem Cell Res 2009, 3:63-70.

31. Du Y, Li X, Yang D, Zhang X, Chen S, Huang K, et al: Multiple molecular pathways are involved in the neuroprotection of GDNF against proteasome inhibitor induced dopamine neuron degeneration in vivo. Exp Biol Med 2008, 233(7):881-890.

32. Wang F, Yasuhara T, Shingo T, Kameda M, Tajiri N, Yuan WJ, et al: Intravenous administration of mesenchymal stem cells exerts therapeutic effects on parkinsonian model of rats: focusing on neuroprotective effects of stromal cell-derived factor-1alpha. BMC Neurosci 2010, 11(52):1-9.

33. Geeraerts T, Deiva K, M'Sika I, Salim H, Hery C, Tardieu M: Effects of SDF1alpha and gp120IIIB on apoptotic pathways in SK-N-SH neuroblastoma cells. Neurosci Lett 2006, 399(1-2):115-120.

34. Shyu WC, Lin SZ, Yen PS, Su CY, Chen DC, Wang HJ, et al: Stromal cellderived factor-1 alpha promotes neuroprotection, angiogenesis, and mobilization/homing of bone marrow-derived cells in stroke rats. J Pharmacol Exp Ther 2008, 324(2):834-849.

35. Zhu B, Xu D, Deng X, Chen Q, Huang Y, Peng H, et al: CXCL12 enhances human neural progenitor cell survival through a CXCR7- and CXCR4mediated endocytotic signaling pathway. Stem Cells 2012, 30(11):2571-2583. 
36. Delcroix GJ, Curtis KM, Schiller PC, Montero-Menei CN: EGF and bFGF pretreatment enhances neural specification and the response to neuronal commitment of MIAMI cells. Differentiation 2010, 80(4-5):213-227.

37. Delcroix GJ, Garbayo E, Sindji L, Thomas O, Vanpouille-Box C, Schiller PC, et al: The therapeutic potential of human multipotent mesenchymal stromal cells combined with pharmacologically active microcarriers transplanted in hemi-parkinsonian rats. Biomaterials 2011, 32(6):1560-1573.

38. Blits B, Kitay BM, Farahvar A, Caperton CV, Dietrich WD, Bunge MB: Lentiviral vector-mediated transduction of neural progenitor cells before implantation into injured spinal cord and brain to detect their migration, deliver neurotrophic factors and repair tissue. Restor Neurol Neurosci 2005, 23(5-6):313-324.

39. Bjorklund A, Kirik D, Rosenblad C, Georgievska B, Lundberg C, Mandel RJ: Towards a neuroprotective gene therapy for Parkinson's disease: use of adenovirus, AAV and lentivirus vectors for gene transfer of GDNF to the nigrostriatal system in the rat Parkinson model. Brain Res 2000, 886(1-2):82-98.

40. Chen L, He DM, Zhang Y: The differentiation of human placenta-derived mesenchymal stem cells into dopaminergic cells in vitro. Cell Mol Biol Lett 2009, 14(3):528-536.

41. Kim YJ, Park HJ, Lee G, Bang OY, Ahn YH, Joe E, et al: Neuroprotective effects of human mesenchymal stem cells on dopaminergic neurons through anti-inflammatory action. Glia 2009, 57(1):13-23.

42. Fischer UM, Harting MT, Jimenez F, Monzon-Posadas WO, Xue H, Savitz SI, et al: Pulmonary passage is a major obstacle for intravenous stem cell delivery: the pulmonary first-pass effect. Stem Cells Dev 2009, 18(5):683-692.

43. Jackson JS, Golding JP, Chapon C, Jones WA, Bhakoo KK: Homing of stem cells to sites of inflammatory brain injury after intracerebral and intravenous administration: a longitudinal imaging study. Stem Cell Res Ther 2010, 1(2):1-12.

44. Stamatovic SM, Keep RF, Andjelkovic AV: Brain endothelial cell-cell junctions: how to "open" the blood brain barrier. Curr Neuropharmacol 2008, 6(3):179-192.

45. Zhang C, Zhou C, Teng JJ, Zhao RL, Song YQ: Multiple administrations of human marrow stromal cells through cerebrospinal fluid prolong survival in a transgenic mouse model of amyotrophic lateral sclerosis. Cytotherapy 2009, 11(3):299-306.

46. Duty S, Jenner P: Animal models of Parkinson's disease: a source of novel treatments and clues to the cause of the disease. Br J Pharmacol 2011, 164(4):1357-1391.

47. Garea-Rodriguez E, Schlumbohm C, Czeh B, Konig J, Helms G, Heckmann C, et al: Visualizing dopamine transporter integrity with iodine-123-FP-CIT SPECT in combination with high resolution MRI in the brain of the common marmoset monkey. J Neurosci Methods 2012, 210(2):195-201.

48. Guo J, Zeng Y, Liang Y, Wang L, Su H, Wu W: Cyclosporine affects the proliferation and differentiation of neural stem cells in culture. Neuroreport 2007, 18(9):863-868.

49. Ibarra A, Correa D, Willms K, Merchant MT, Guizar-Sahagun G, Grijalva I, et al: Effects of cyclosporin-A on immune response, tissue protection and motor function of rats subjected to spinal cord injury. Brain Res 2003, 979(1-2):165-178.

50. Palladini G, Caronti B, Pozzessere G, Teichner A, Buttarelli FR, Morselli E, et al: Treatment with cyclosporine A promotes axonal regeneration in rats submitted to transverse section of the spinal cord-II-Recovery of function. J Hirnforsch 1996, 37(1):145-153.

51. Bouchez G, Sensebe L, Vourc'h P, Garreau L, Bodard S, Rico A, et al: Partial recovery of dopaminergic pathway after graft of adult mesenchymal stem cells in a rat model of Parkinson's disease. Neurochem Int 2008, 52(7):1332-1342

52. Park HJ, Lee PH, Bang OY, Lee G, Ahn YH: Mesenchymal stem cells therapy exerts neuroprotection in a progressive animal model of Parkinson's disease. J Neurochem 2008, 107(1):141-151.

53. Danielyan L, Schafer R, von Ameln-Mayerhofer A, Bernhard F, Verleysdonk S, Buadze $M$, et al: Therapeutic efficacy of intranasally delivered mesenchymal stem cells in a rat model of Parkinson disease. Rejuvenation Res 2011, 14(1):3-16.

54. Chao YX, He BP, Tay SS: Mesenchymal stem cell transplantation attenuates blood brain barrier damage and neuroinflammation and protects dopaminergic neurons against MPTP toxicity in the substantia nigra in a model of Parkinson's disease. J Neuroimmunol 2009, 216(1-2):39-50.

55. Sadan O, Bahat-Stromza M, Barhum Y, Levy YS, Pisnevsky A, Peretz $H$, et al: Protective effects of neurotrophic factor-secreting cells in a 6-OHDA rat model of Parkinson disease. Stem Cells Dev 2009, 18(8):1179-1190.
56. Levy YS, Bahat-Stroomza M, Barzilay R, Burshtein A, Bulvik S, Barhum Y, et al: Regenerative effect of neural-induced human mesenchymal stromal cells in rat models of Parkinson's disease. Cytotherapy 2008, 10(4):340-352.

57. Cova L, Armentero MT, Zennaro E, Calzarossa C, Bossolasco P, Busca G, et al: Multiple neurogenic and neurorescue effects of human mesenchymal stem cell after transplantation in an experimental model of Parkinson's disease. Brain Res 2010, 1311:12-27.

58. Blandini F, Cova L, Armentero MT, Zennaro E, Levandis G, Bossolasco P, et al: Transplantation of undifferentiated human mesenchymal stem cells protects against 6-hydroxydopamine neurotoxicity in the rat. Cell Transplant 2010, 19(2):203-217.

59. Zhu Q, Ma J, Yu L, Yuan C: Grafted neural stem cells migrate to substantia nigra and improve behavior in Parkinsonian rats. Neurosci Lett 2009, 462(3):213-218.

60. Bjugstad KB, Teng YD, Redmond DE Jr, Elsworth JD, Roth RH, Cornelius SK, et al: Human neural stem cells migrate along the nigrostriatal pathway in a primate model of Parkinson's disease. Exp Neurol 2008, 211(2):362-369.

61. Ramos-Moreno T, Castillo CG, Martinez-Serrano A: Long term behavioral effects of functional dopaminergic neurons generated from human neural stem cells in the rat 6-OH-DA Parkinson's disease model. Effects of the forced expression of BCL-X(L). Behav Brain Res 2012, 232(1):225-232.

62. Park HJ, Bang G, Lee BR, Kim HO, Lee PH: Neuroprotective effect of human mesenchymal stem cells in an animal model of double toxin-induced multiple system atrophy parkinsonism. Cell Transplant 2011, 20(6):827-835.

63. Lee PH, Kim JW, Bang OY, Ahn YH, Joo IS, Huh K: Autologous mesenchymal stem cell therapy delays the progression of neurological deficits in patients with multiple system atrophy. Clin Pharmacol Ther 2008, 83(5):723-730.

64. Venkataramana NK, Kumar SK, Balaraju S, Radhakrishnan RC, Bansal A, Dixit A, et al: Open-labeled study of unilateral autologous bone-marrow-derived mesenchymal stem cell transplantation in Parkinson's disease. Translational research : the journal of laboratory and clinical medicine 2010, 155(2):62-70.

65. Venkataramana NK, Pal R, Rao SA, Naik AL, Jan M, Nair R, et al: Bilateral transplantation of allogenic adult human bone marrow-derived mesenchymal stem cells into the subventricular zone of Parkinson's disease: a pilot clinical study. Stem Cells Int 2012, ID931902:1-12.

66. Dimmeler S, Leri A: Aging and disease as modifiers of efficacy of cell therapy. Circ Res 2008, 102(11):1319-1330.

67. Li TS, Kubo M, Ueda K, Murakami M, Mikamo A, Hamano K: Impaired angiogenic potency of bone marrow cells from patients with advanced age, anemia, and renal failure. J Thorac Cardiovasc Surg 2010, 139(2):459-465.

68. Koh SH, Baik W, Noh MY, Cho GW, Kim HY, Kim KS, et al: The functional deficiency of bone marrow mesenchymal stromal cells in ALS patients is proportional to disease progression rate. Exp Neurol 2012, 233(1):472-480.

69. Zhang Z, Wang X, Wang S: Isolation and characterization of mesenchymal stem cells derived from bone marrow of patients with Parkinson's disease. In Vitro Cell Dev Biol Anim 2008, 44(5-6):169-177.

70. Somoza R, Juri C, Baes M, Wyneken U, Rubio FJ: Intranigral transplantation of epigenetically induced BDNF-secreting human mesenchymal stem cells: implications for cell-based therapies in Parkinson's disease. Biol Blood Marrow Transplant 2010, 16(11):1530-1540.

71. Ourednik J, Ourednik V, Lynch WP, Schachner M, Snyder EY: Neural stem cells display an inherent mechanism for rescuing dysfunctional neurons. Nat Biotechnol 2002, 20(11):1103-1110

72. Chen X, Liu W, Guoyuan Y, Liu Z, Smith S, Calne DB, et al: Protective effects of intracerebral adenoviral-mediated GDNF gene transfer in a rat model of Parkinson's disease. Parkinsonism Relat Disord 2003, 10(1):1-7.

73. McCluskey L, Campbell S, Anthony D, Allan SM: Inflammatory responses in the rat brain in response to different methods of intra-cerebral administration. J Neuroimmunol 2008, 194(1-2):27-33.

74. Perry VH, Bell MD, Brown HC, Matyszak MK: Inflammation in the nervous system. Curr Opin Neurobiol 1995, 5(5):636-641.

doi:10.1186/2047-9158-2-13

Cite this article as: de Munter et al: Cell based therapy in Parkinsonism.

Translational Neurodegeneration 2013 2:13. 